\title{
Molecularly Imprinted Polymer as a Sorbent for Alpha Mangostin
}

\author{
Neena Zakia ${ }^{1,2}$, Handajaya Rusli $^{1}$, M Ali Zulfikar ${ }^{1}$, and M Bachri Amran ${ }^{1}$ \\ \{neena.zakia.fmipa@um.ac.id, handajaya@chem.itb.ac.id, zulfikar@chem.itb.ac.id, \\ amran@chem.itb.ac.id\} \\ Division of Analytical Chemistry, Faculty of Mathematics and Natural Sciences, Institut Teknologi \\ Bandung ${ }^{1}$ \\ Department of Chemistry, Faculty of Mathematics and Natural Sciences, Universitas Negeri Malang ${ }^{2}$
}

\begin{abstract}
Alpha mangostin is a major component in the mangosteen rind, which has activity as an antioxidant, antitumor, and anticancer. The aim of this study is to synthesize molecularly imprinted polymer (MIP) for alpha mangostin adsorption. The MIP was prepared by bulk polymerization with alpha mangostin as template, methacrylic acid as the monomer, ethylene glycol dimethacrylate as the crosslinker, and benzoyl peroxide as the initiator, and acetonitrile as porogenic solvent. A molar ratio of the template:monomer:crosslinker is 1:2:10. The surface morphology of MIP was visualized by scanning electron microscopy (SEM). The synthesized MIP was characterized by infrared spectroscopy. It showed that wavelength numbers were 2957, 1603, and $1456 \mathrm{~cm}^{-}$ ${ }^{1}$ assigned to characteristic vibration of a $\mathrm{C}-\mathrm{H}$ bond, a chelated carbonyl group, and a methoxy group, respectively, which indicated the presence of template molecule. The adsorption capacity of MIP for alpha mangostin was $12,17 \mathrm{mg} / \mathrm{g}$ with the imprinting factor 1,2 .
\end{abstract}

Keywords: alpha mangostin, molecularly imprinted polymer, adsorption

\section{Introduction}

Mangosteen (Garcinia mangostana L.) is an Indonesian commodity with a large abundance. Besides consuming the fruit, mangosteen is also used as traditional medicine. This is due to the pharmacological properties of the compounds in the mangosteen. The benefits of mangosteen for healing diseases such as fever, diarrhea, dysentery, and skin diseases [1]. Bioactive compounds in mangosteen are found in its pericarp [1-4]. Chemical compounds contained in the pericarp are xanthon and its derivatives, benzo-phenones, flavonoids, anthosianin, and tannins $[5,6]$. The greatest abundance of xanthon in mangosteen is alpha mangostin compound $[1,4]$.

Each bioactive compound in mangosteen pericarp has different benefits. Alpha mangostin compounds are known to have activities as antibacterial, anti-inflammatory, antioxidant, hypoallergenic, antitumor, and anticancer $[1,7]$. To determine one of the compounds in a complex matrix, several stages of analysis are carried out such as isolation, clean-up, and preconcentration $[8,9]$. The cleanup procedure performed is solid-phase extraction (SPE). In recent years, molecularly imprinted polymer (MIP) is being extensively employed as the sorbent of the solid phase extraction (MISPE). The use of MIP in SPE provides an advantage to clean 
up the interfering components from complex matrices and enrich the target analyte (preconcentration) [10,11].

Molecularly imprinted polymer (MIP) is polymeric material which has a high affinity and selectivity toward a particular target analyte or a group of the structurally related compound. The MIP has a cavity as a recognition site that results from the interaction between the template molecule and functional monomer [12]. MIP material for the purposes of clean-up in analysis has been widely applied to natural products [6, 12, 13], drugs [14], food [15], and environmental samples [16]. In this study, the synthesis of MIP will be carried out for alpha mangostin target compounds. This work describes the preparation of molecularly imprinted polymer by bulk polymerization with methacrylic acid as the monomer, ethylene glycol dimethacrylate as the crosslinker, and benzoyl peroxide as the initiator and acetonitrile as porogenic solvent. Characterization of this polymer was carried out by Fourier transform infrared (FTIR) spectroscopy and surface morphology by scanning electron microscopy (SEM). Evaluation of binding capacity was investigated by the batch experiment. Finally, the MIP was used as a sorbent for alpha mangostin.

\section{Experimental}

Alpha mangostin and ethylene glycol dimethacrylate (EGDMA) were purchased from Sigma Aldrich. Methacrylic acid (MAA), benzoyl peroxide (BPO), acetonitrile, methanol, and acetic acid were provided by Merck. Distilled water was filtered by an Easypure RF Barnstead. FTIR spectroscopic measurements were performed on Fourier transform infrared spectrophotometer IR Prestige-21 Shimadzu with $\mathrm{KBr}$ pellet method. Analysis of surface morphology by Scanning Electron Microscope (SEM) FEI type INSPECT-S50. UV-Vis analysis was performed using UV-Vis spectrometry Agilent technologies HP 8453. Measurement of adsorption capacity by HPLC Infinity Agilent Tech 1260 Series.

The molecularly imprinted polymer (MIP) and non-molecular imprinted polymer (NIP) were prepared by bulk polymerization. The MIP was synthesized by dissolving $0,5 \mathrm{mmol}$ of a template (alpha mangostin) and $1 \mathrm{mmol}$ methacrylic acid (MAA) as the monomer in $10 \mathrm{~mL}$ acetonitrile in a glass tube. The mixture was homogenized for 24 hours for prapolymerization. Then, 5 mmol EGDMA and $375 \mathrm{mg}$ of benzoyl peroxide (BPO) were added into the solution. The mixture was purged with nitrogen gas for 10 minutes to remove oxygen. Polymerization was performed in an oil bath at $60^{\circ} \mathrm{C}$ for 8 hours. After polymerization, the template was extracted using solvent extraction to remove the alpha mangostin as a template with methanol: acetic acid (9:1) for 24 hours. The amount of template remaining was monitored by UV spectrometry. Non-imprinted polymer (NIP) was prepared similar to MIP with the absence of a template molecule. The molecularly imprinted polymer was dried, ground, and sieved to obtain sizes 60 mesh

Molecularly imprinted polymer was characterized using by FTIR instrument and scanning electron microscopy. The IR spectrum was recorded in order to identify the functional groups of the MIP. The method used was KBr pellet. The surface morphology of the MIP was observed by scanning electron microscope.

Evaluation of binding ability was using batch rebinding experiments. $25 \mathrm{mg}$ of MIP sorbent was dissolved in $10 \mathrm{~mL}$ of $25 \mathrm{mg} / \mathrm{L}$ alpha mangostin standard solution (in methanol: water 1:1) and incubated overnight by shaking in $150 \mathrm{rpm}$ at room temperature. Then the mixture was centrifuged and the supernatant was measured by using high-performance liquid 
chromatography. The mobile phase was methanol: water $(95: 5, \mathrm{v} / \mathrm{v})$, with a flow rate of 1 $\mathrm{mL} / \mathrm{min}$. $20 \mu \mathrm{L}$ of the supernatant was taken out for HPLC analysis to measure the amount of alpha mangostin remained. The adsorption capacity of alpha mangostin was calculated by dividing the amount of analyte remain in the solution by the weight of the sorbent. The imprinting factor (IF) was calculated from the equation (1)

$$
\mathrm{IF}=\mathrm{Q}_{\mathrm{MIP}} / \mathrm{Q}_{\mathrm{NIP}}
$$

where $\mathrm{Q}_{\mathrm{MIP}}$ and $\mathrm{Q}_{\mathrm{NIP}}$ are the total binding amount of MIP and NIP, respectively

\section{Results and Discussion}

Molecularly imprinted polymer (MIP) for alpha mangostin was synthesized and prepared in this work. Synthesis of MIP was carried out in bulk polymerization by a non-covalent approach. MIP was prepared using alpha mangostin as a template, methacrylic acid as the monomer, ethylene glycol dimethacrylate as the crosslinker, and benzoyl peroxide as the initiator and acetonitrile as porogenic solvent.

The alpha mangostin molecule is an acidic organic compound with $\mathrm{pKa}_{1}$ of 3.89 which containing three phenolic hydroxyl $-\mathrm{OH}$ functional groups in the structure. It can form hydrogen-bonding interactions with the monomer to produce a stable template-monomer complex. Methacrylic acid was used as the monomer because its carboxyl group is the most common hydrogen bonding and acidic functional group in molecular imprinting cross-linked with ethylene glycol dimethacrylate (EGDMA). Methacrylic acid was selected as a functional monomer due to it can not only form hydrogen bonds with the template molecule but also easiness for released to form a cavity. Interaction between functional monomer and template will produce a recognition cavity that has selective binding sites that appropriate for the target molecule. In this study, a molar ratio between the template:monomer is 1:2. In some studies, the most favorable ratio of template:monomer is 1:4 for most polymers [6, 17].

However, there also studies that reported the best performance was shown molar ratio template: monomer in 1:2 using methacrylic acid as a monomer [17].

In order to produce the cavity with a selective recognition site, a crosslinker is required for the stability of the monomer-template complex during the imprinting process. The amount of crosslinker should be enough to maintain the stability of the recognition sites and control the morphology of the MIP. Ethylene glycol dimethacrylate is a crosslinker that commonly used with methacrylic acid due to it is proven to form good mechanical stability of polymer [18].

After removal of the template molecule through soxhlet extraction from polymer, it will leave a cavity which has an active site and shape for rebinding template molecule. In order to verify that the retention of alpha mangostin was due to molecular recognition and not due to nonspecific binding, control non-imprinted polymer (NIP) was prepared using the same procedure without the template.

Imprinting process occurs due to the complexity between the functional monomer and the template to form hydrogen bonds. It can be identified from the FTIR spectra was shown in Figure 1. 


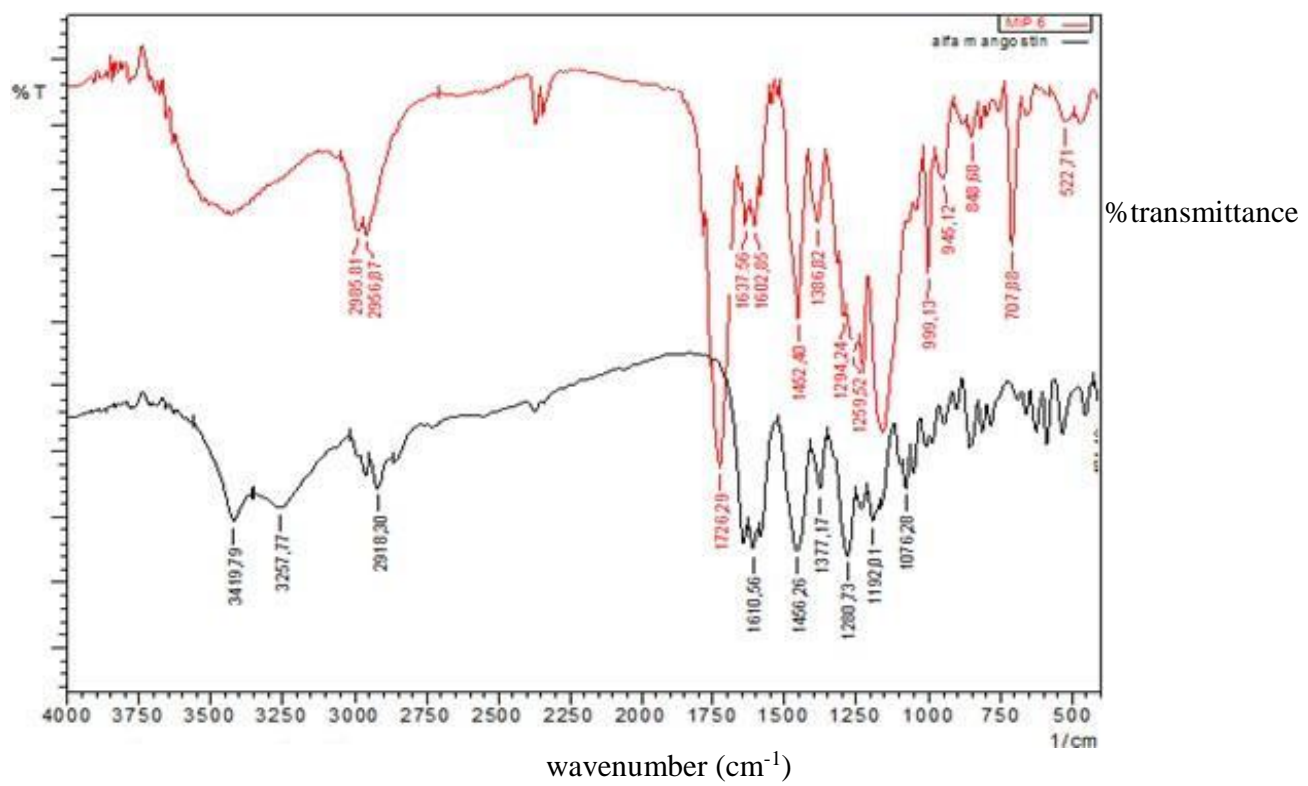

Fig. 1. FTIR spectra for (a) MIP and (b) alpha mangostin.

The broadband characteristic at $3300-3600 \mathrm{~cm}^{-1}$ indicates the formation of intermolecular hydrogen bonding between alpha mangostin and methacrylic acid. Based on FTIR spectra that confirmed the presence of functional groups from template molecule as shown in Table 1.

The scanning electron microscope (SEM) was used to examine MIP morphologically. From the SEM micrographs in Figure 2, polymers exhibit smooth. The morphology of MIP after leaching was more hollow than MIP before leaching. This was probably due to the fact that soxhlet extraction was succeeded to remove the template to form the cavity. The MIP after leaching has a morphology that similar to non imprinted polymer.

Table 1. Wavenumber data for FTIR spectra of alpha mangostin imprinted polymer and alpha mangostin.

\begin{tabular}{lcc}
\hline \multicolumn{1}{c}{ Functional Group } & \multicolumn{2}{c}{ Wavenumber $\left(\mathrm{cm}^{-1}\right)$} \\
\cline { 2 - 3 } & alpha mangostin & MIP \\
\hline O-H stretch & 3420 & $3300-3600$ \\
C-H stretch & 2918 & 2957 \\
C=O stretch & 1611 & 1603 \\
C=C aromatic stretch & 1456 & 1462 \\
symmetric bending vibration of $-\mathrm{CH}_{3}$ & 1377 & 1387 \\
C-O stretch & 1281 & 1260 \\
\hline
\end{tabular}



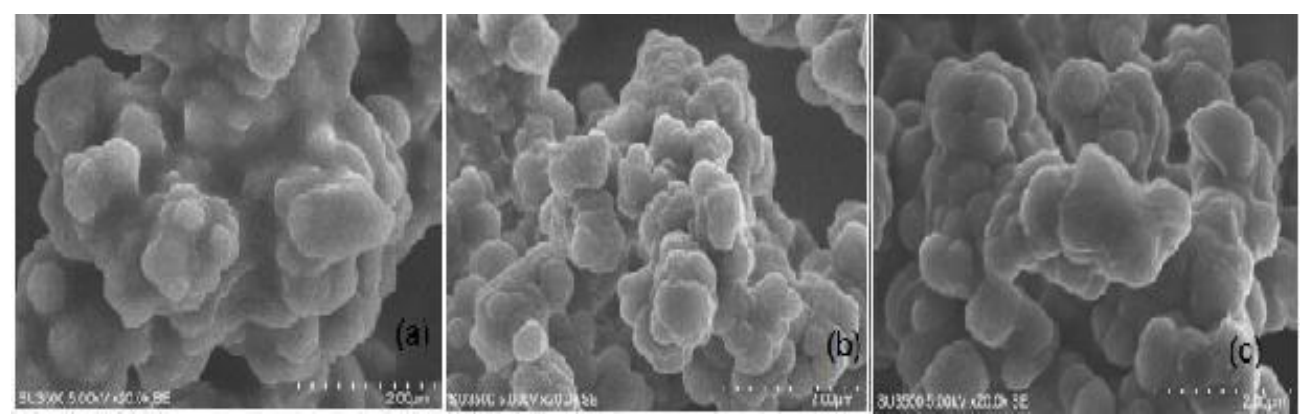

Fig. 2. SEM micrograph of polymer: (a) MIP before leaching, (b) MIP after leaching, (c) NIP.

Evaluation of the binding ability of MIP was done by using the batch experiment. The binding analysis was carried out by incubating overnight of MIP in alpha mangostin solution using methanol:aquaeus (1:1) solvent. The calculated binding capacity of MIP was 12,17 mg alpha mangostin/g MIP. These results confirmed that MIP can adsorp the alpha mangostin. The adsorption capacity of the control polymer was $10,07 \mathrm{mg}$ alpha mangostin/g NIP. We found that the binding capacity of the alpha mangostin-imprinted polymer was better than the nonimprinted polymer. It can be observed that the MIP can bind to alpha mangostin greater than that of the control polymer which is indicated by the imprinting factor is 1.2. These results indicated that MIP has a cavity as a recognition site for alpha mangostin than the control polymer. To further improve the MIP binding performance, method optimization is needed to obtain the maximum adsorption capacity of the synthesized MIP.

\section{Conclusion}

Molecularly imprinted polymer for alpha mangostin was successfully synthesized by bulk polymerization. From the experimental results, characterization by IR spectroscopy showed that the synthesized MIP indicated the presence of functional groups of the template molecules. SEM analysis shows that there are differences in MIP before and after removal of the template, which indicates that there probably a cavity as a recognition site. The synthesized MIP was shown good binding for alpha mangostin as compared to the control polymer. Furthermore, this MIP has a good chance to be used as a functional material for alpha mangostin adsorption.

Acknowledgements. The author is very grateful to Research, Technology, and Higher Education Ministry for their financial support through Doctoral Dissertation Research (PDD) Grant 2019.

\section{References}

[1] Pedraza-Chaverri, J., Cárdenas-Rodríguez, N., Orozco-Ibarra, M, and Pérez-Rojas, J. M.: Review: Medicinal properties of mangosteen (Garcinia mangostana). Food and Chemical Toxicology. Vol. 46, pp. 3227-3239 (2008) 
[2] Jung, H-A., Su, B-N., Keller, W.J., Mehta, R.G., and Kinghorn, A.D.: Antioxidant xanthones from the pericarp of Garcinia mangostana (mangosteen). Journal of Agricultural and Food Chemistry. Vol. 54, pp. 2077-2082 (2006)

[3] Al-Massarani, S.M., El Gamal, A.A., Al-Musayeib, N.M., Mothana, R.A., Basudan, O.A., AlRehaily, A.J., Farag, M., Assaf, M.H., El Tahir, K.H., and Maes, L.: Phytochemical, antimicrobial, and antiprotozoal evaluation of Garcinia mangostana pericarp and $\alpha$-mangostin, its major xanthone derivative. Molecules. Vol. 18, pp. 10599-10608 (2013)

[4] Yoshimura, M., Ninomiya, K., Tagashira, Y., Maejima, K., Yoshida, T., and Amakura, Y.: Polyphenolic constituents of the pericarp of mangosteen (Garcinia mangostana L.). Journal of Agricultural and Food Chemistry. Vol. 63(35), pp. 7670-7674 (2015)

[5] Mahabusarakam, W. and Wiriyacithitra, P.: Chemical constituents of Garnicia mangostana. Journal of Natural Products. Vol. 50(3), pp. 474-478 (1987)

[6] Piacham, T., Isarankura-Na-Ayudhya, C., and Prachayasittikul, V.: Quercetin-imprinted polymer for anthocyanin extraction from mangosteen pericarp. Material Science and Engineering. Vol. 51, pp. 127-131 (2015)

[7] Akao, Y., Nakagawa, Y., Iinuma, M., and Nozawa, Y.: Review: Anticancer effects of xanthones from pericarps of mangosteen. International Journal of Molecular Sciences. Vol. 9, pp. 355-370 (2008) [8] Zuloagaa, O., Navarroa, P., Bizkarguenagaa, E., Iparraguirrea, A., Vallejoa, A., Olivaresa, M., and Prietoa, A.: Overview of extraction, clean-up and detection techniques for the determination of organic pollutants in sewage sludge: A review. Analytica Chimica Acta. Vol. 736, pp. 7-29 (2012)

[9] Schuhmacher, R., Krska, R., Grasserbauer, M., Edinger, W., and Lew, H.: Immuno-affinity columns versus conventional clean-up: a method-comparison study for the determination of zearalenone in corn. Fresenius Journal of Analytical Chemistry. Vol. 360, pp. 241-245 (1998)

[10] Żwir-Ferenc, A. and Biziuk, M.: Review: Solid Phase Extraction Technique - Trends, Opportunities and Applications. Polish Journal of Environmental Studies. Vol. 15, Issue 5, pp. 677690 (2006)

[11] Lok, C. M. and Son, R.: Application of molecularly imprinted polymers in food sample analysis - a perspective: Review Article. International Food Research Journal. Vol. 16, pp.127-140 (2009)

[12] Song, X. J.L., Wanga, J., and Chen, L.: Quercetin molecularly imprinted polymers: preparation, recognition characteristics and properties as sorbent for solid-phase extraction. Talanta. Vol. 80, pp 694-702 (2009)

[13] Suwanwong, Y., Kulkeratiyut, S., Prachayasittikul, V., and Boonpangrak, S.: Effects of polymerization methods and functional monomers on curcumin imprinted polymer properties. Separation Science and Technology. Vol. 49, pp. 1086-1095 (2014)

[14] Kartasasmita, R.E., Hasanah, A.N., and Ibrahim, S.: Synthesis of selective molecularly imprinted polymer for solid-phase extraction of glipizide by using a pseudo-template. Journal of Chemical and Pharmaceutical Research. Vol. 5(10), pp. 351-355 (2013)

[15] Sadeghi, S. and Jahani, M.: Selective solid-phase extraction using molecular imprinted polymer sorbent for the analysis of florfenicol in food samples. Food Chemistry. Vol. 141, pp. 1242-1251 (2013) [16] Zakaria, N.D., Haron, N.A.Y.J., and Abdullah, A.H.: Synthesis and Evaluation of a Molecularly Imprinted Polymer for 2,4-Dinitrophenol. International Journal of Molecular Sciences. Vol. 10, pp. 354-365 (2009)

[17] Guć, M. And Schroeder, G.: The Molecularly Imprinted Polymers. Influence of Monomers on The Properties of Polymers - A Review. World Journal of Research and Review (WJRR). Vol. 5 (6), pp. 36-47 (2017)

[18] Yan, H., Jin, L., and Row, K.H.: Special selectivity of molecularly imprinted monolithic stationary phase. Journal of Liquid Chromatography \& Related Technology. Vol. 28, pp. 3147-3155 (2005) 